\title{
SALUD Y CRECIMIENTO ECONÓMICO: INFLUENCIAS TEÓRICAS Y VINCULACIONES EMPÍRICAS
}

\section{HEALTH AND ECONOMIC GROWTH: THEORETICAL INFLUENCES AND EMPIRICAL VINCULATIONS}

\author{
Pablo Daniel Monterubbianesi *
}

\section{RESUMEN}

El objetivo del presente artículo consiste en describir los principales canales de influencia del status de salud sobre el crecimiento económico. Asimismo, se realiza un análisis de estadística comparativa sobre la situación entre los diferentes países y regiones, respecto a las variables salud e ingreso. Finalmente, se intenta verificar desde el punto de vista empírico, mediante la técnica de agrupamiento por tramos, la existencia de una estrecha relación entre estas dimensiones, considerando la situación relativa de los diferentes países del mundo.

PALABRAS CLAVE: SALUD * CRECIMIENTO ECONÓMICO * INGRESO * BIENESTAR SOCIAL * AGRUPAMIENTO POR TRAMOS

\section{ABSTRACT}

The aim of this article is to describe the main channels of influence of health status on economic growth. Also, to conduct a comparative statistical analysis on the situation between the different countries and regions about health and income. Finally, attempt to verify from the empirical point of view, using clustering technique in installments, the existence of a close relationship these dimensions, considering the relative situation of different countries.

KEYWORDS: HEALTH * ECONOMIC GROWTH * INCOME * SOCIAL WELFARE * CLUSTERING TECHNIQUE IN INSTALLMENTS

\footnotetext{
* Instituto de Investigaciones Económicas y Sociales del Sur (IIESS), Argentina.
} pmonteru@uns.edu.ar 


\section{INTRODUCCIÓN}

Habitualmente, la teoría económica reconocía a la educación como componente principal y único del capital humano. Sin embargo, con el correr del tiempo, la salud se ha ido considerando como un componente relevante del mismo. Así, considerando el efecto del capital humano sobre el crecimiento económico, resulta de interés el análisis del efecto de la salud sobre el crecimiento y de este modo, sobre la calidad de vida de las diferentes sociedades (Monterubbianesi, 2010).

De esta forma, en la evolución de la teoría del crecimiento económico, se han realizado diferentes modelos en los cuales, la salud juega un rol fundamental como determinante del crecimiento. Estos modelos normalmente trabajan empleando las herramientas teóricas habituales (generaciones superpuestas y optimización intertemporal) e incluyen la salud como un elemento relevante dentro del modelo. En este contexto, es posible destacar los aportes de Ehrlich y Lui (1991), Barro (1996), Kalemli-Ozcan, Ryder y Weil (2000), Howitt (2005) y Van Zon y Muysken (2005). Así, el aporte de estos autores permite identificar las diferentes vías por las cuales la salud afecta al crecimiento económico.

El análisis de estos modelos muestra una estrecha relación entre el status de salud y el crecimiento económico. La mencionada relación puede abordarse desde la perspectiva del análisis estadístico. Sin embargo, la asociación entre estas dimensiones no es inmediata ni es algo estático. Esta relación del status de salud con el crecimiento, se traduce en el nivel de ingreso, al ser la tasa de crecimiento la variación temporal del mismo. De esta forma, para comprender la relación entre salud y crecimiento en un momento temporal, resulta adecuado vincular la dimensión salud con el nivel de ingreso y no con la tasa de crecimiento.

La técnica de agrupamiento por tramos permite identificar las posiciones relativas de los distintos países, en cuanto a salud e ingreso (London, Temporelli y Monterubbianesi, 2009) y la proximidad de la situación de estos países respecto a las mencionadas dimensiones.

De este modo, el objetivo del presente artículo consiste en describir los principales canales de influencia del status de salud sobre el crecimiento económico. Asimismo, se realiza un análisis de estadística comparativa sobre la situación entre los diferentes países y regiones respecto a salud e ingreso. Finalmente, se intenta verificar desde el punto de vista empírico, mediante la técnica de agrupamiento por tramos, la existencia de una estrecha relación entre estas dimensiones, tal como lo indican los modelos desarrollados en la segunda sección, considerando la situación relativa de los diferentes países del mundo.

El trabajo se encuentra estructurado de la siguiente manera. En la segunda sección, se presentan los diferentes canales de influencia de la salud sobre el crecimiento propuestos por la teoría económica. Posteriormente, se considera la situación relativa de los diferentes continentes en cuanto a salud e ingreso, analizando el grado de desigualdad en el valor de los indicadores para cada una de estas regiones. En la cuarta sección, se considera la situación de estas dimensiones en los distintos países del mundo, aplicando la técnica de agrupamiento por tramos con el objeto de evaluar el grado de proximidad de la situación relativa de cada país respecto a estos indicadores. Por último, en la quinta sección se presentan las principales conclusiones.

\section{SALUD Y CRECIMIENTO ECONÓMICO: CANALES DE INFLUENCIA}

Diferentes autores han desarrollado contribuciones de forma tal de incorporar a la salud dentro de la teoría del crecimiento económico como un determinante fundamental del mismo. A continuación, se presentan los aportes realizados por los autores, identificando los distintos canales de influencia de la salud sobre el crecimiento (Monterubbianesi, 2011).

Antes de introducir la presentación de los aportes, resulta necesario aclarar el concepto de status de salud utilizado por los autores. En estos modelos, este status es considerado un stock equivalente a la educación, siendo ambos componentes del capital humano. De esta forma, la salud queda definida como una dimensión que en los análisis empíricos, puede aproximarse mediante diferentes indicadores 
tales como, la esperanza de vida al nacer o la tasa de mortalidad infantil.

Un primer aporte en la consideración de esta relación se remonta a Ehrlich y Lui (1991), quienes construyen un modelo de generaciones superpuestas donde, a través del capital humano, las diferentes generaciones se encuentran relacionadas y se da lugar al proceso de crecimiento económico. En este modelo, los padres invierten en sus hijos para que luego cuiden de ellos en su vejez, generándose un proceso de optimización intertemporal que maximiza las oportunidades de crecimiento.

En este marco, el rol de la salud surge a partir de las probabilidades de sobrevivir a la adultez y a la adultez mayor que tiene un niño. Incrementos en los parámetros que miden la longevidad, es decir, en estas probabilidades se da lugar a un incremento en la inversión en capital humano $y$ de esta forma, en la tasa de crecimiento de largo plazo. Así, a partir de este modelo de generaciones superpuestas, los autores logran modelizar la existencia de un rol fundamental de la salud en el proceso de crecimiento económico.

Por su parte, Barro (1996) realiza también un aporte al análisis de la relación entre salud y crecimiento económico a partir de la presentación de una versión ampliada del modelo neoclásico. La contribución principal se basa en que la tasa de crecimiento depende no solo de los inputs tradicionales, sino también de la salud de los trabajadores. En el modelo, supone también la existencia de familias productoras que maximizan su utilidad a lo largo del tiempo.

El autor identifica dos vías diferentes por las cuales la salud afecta al crecimiento económico. Por un lado, existe un impacto directo de la salud en la productividad, en el sentido que un incremento en el status de salud eleva la productividad de los trabajadores, impacto que puede observarse por la inclusión del stock de salud como un factor de producción. Por otro lado, existe también un efecto indirecto, el cual se relaciona con que una mejora en la salud reduce las tasas de mortalidad y enfermedad, por lo tanto, disminuye la tasa de depreciación del capital humano, tanto de la educación como de la salud. De esta forma, un incremento en el status de salud eleva la demanda de capital humano, generándose un efecto indirecto positivo sobre la productividad.

Kalemli-Ozcan, Ryder y Weil (2000) construyen un modelo que postula la existencia de una conducta de ciclo de vida del individuo. La riqueza del individuo al momento de su nacimiento es 0 y declina mientras está en el período educativo, alcanzándose un mínimo en el momento en el que comienza a trabajar. Durante sus primeros años de trabajo, los individuos pagan sus deudas y comienzan a tener una riqueza positiva. Aunque los salarios se mantienen constantes durante la vida del trabajador, el consumo está creciendo. El sendero de consumo que definimos requiere que el nivel de riqueza se incremente a lo largo de la vida del individuo. De este modo, el modelo supone que el individuo acumula riqueza con el objetivo de aprovechar la diferencia entre la tasa de interés $y$ su tasa subjetiva de descuento.

La salud es introducida en este ciclo de vida a partir del hecho de que afecta la productividad. Por lo tanto, este análisis lo que indica es que una caída en la probabilidad de muerte a lo largo de la vida del individuo, aumenta su nivel de escolaridad y su stock de capital humano, generándose un proceso de crecimiento en la economía. De este modo, es posible mediante el modelo, analizar la relación entre salud y crecimiento económico.

Un cuarto aporte lo realiza Peter Howitt (2005), quien presenta un modelo basado en el concepto schumpeteriano de progreso técnico, es decir, empleando la idea de destrucción creativa, donde una nueva innovación desplaza a la anterior. A partir de una serie de modificaciones en este modelo, es posible incorporar la salud por medio de una serie de parámetros. En base a esta dinámica, se definen dos grupos de países, aquellos que crecerán a la tasa de crecimiento de la tecnología mundial y aquellos que quedarán rezagados respecto a dicha tecnología, creciendo en menor medida.

Respecto a la vinculación entre salud y crecimiento económico, Howitt (2005) define entonces seis canales de influencia, los cuales se reflejan en los parámetros del modelo analizado. 
La primera de estas vías se relaciona con la eficiencia en la productividad, la cual se basa en la idea de que trabajadores más saludables serán más productivos. Un segundo canal por la cual la salud afecta el crecimiento ocurre a través de la esperanza de vida, que influirá en la tasa de depreciación de las habilidades, modificando así a la tasa de crecimiento de las mismas. La tercera y cuarta vías surgen del hecho de que individuos con un mejor status de salud, tendrán una mayor capacidad de aprendizaje y de adaptabilidad a los cambios, mientras que una quinta vía se relaciona con que un mejor status de salud, principalmente durante la niñez, hace que las personas sean más creativas. El último camino mencionado por el autor se vincula con la distribución del ingreso. Un mayor stock de salud afectará al sector de la población más desprotegido, disminuyendo la desigualdad, elevando el nivel de escolaridad y de esta forma, la tasa de crecimiento.

Por último, se destaca la contribución que presentan Van Zon y Muysken (2005), quienes a partir del modelo de Lucas (1988), desarrollan una versión modificada del mismo, incorporando a la salud como determinante del crecimiento económico. Para ello, distinguen en primer lugar dos estados de salud que la población puede tener: los habitantes pueden pertenecer a la población saludable o a la población no saludable. Cualquier individuo de la población puede cambiar de estado por causas externas o bien, por cuestiones relacionadas con la salud. La conducta del modelo puede establecerse sosteniendo que todos los individuos nacen saludables y solo los individuos saludables se reproducen. Adicionalmente, las personas saludables no mueren sin antes enfermarse.

En resumen, se sostiene que el aporte realizado por los autores permite, mediante una serie de modificaciones al modelo de Lucas (1988), establecer la relación entre el status de salud y el crecimiento económico, la cual es posible definir a través de dos vías. Por un lado, una caída en el nivel de salud promedio de la población puede causar una caída en la oferta de trabajo efectivo. Por otro lado, la generación de salud toma recursos que tienen usos alternativos, es decir destinados a la producción.

De este modo, se identifican los canales presentes en la literatura a través de los cuales la salud afecta al crecimiento económico. La tabla 1 muestra entonces los diferentes canales y a los autores que han identificado cada uno de ellos.

TABLA 1

CANALES DE INFLUENCIA DE LA SALUD SOBRE EL CRECIMIENTO ECONÓMICO

\begin{tabular}{l|l}
\hline CANAL & \multicolumn{1}{l}{ AUTORES } \\
\hline $\begin{array}{l}\text { Mayor status de salud, mayor productividad, mayor } \\
\text { tasa de crecimiento. }\end{array}$ & $\begin{array}{l}\text { Barro (1996), Howitt (2005), Van Zon y Muysken } \\
(2005) .\end{array}$ \\
$\begin{array}{l}\text { Mayor status de salud, menor tasa de depreciación, } \\
\text { mayor horizonte de vida, mayor inversión en capital } \\
\text { humano, mayor tasa de crecimiento. }\end{array}$ & $\begin{array}{l}\text { Ehrlich y Lui (1991), Barro (1996), Kalemly-Ozcan, } \\
\text { Ryder Weil (2000), Howitt (2005). }\end{array}$ \\
$\begin{array}{l}\text { Mayor status de salud, mayor capacidad de } \\
\text { aprendizaje, mayor crecimiento. }\end{array}$ & Howitt (2005). \\
$\begin{array}{l}\text { Mayor status de salud, mayor creatividad, mayor } \\
\text { crecimiento. }\end{array}$ & Howitt (2005). \\
$\begin{array}{l}\text { Mayor status de salud, menor desigualdad, mayor } \\
\text { escolaridad, mayor crecimiento. }\end{array}$ & Howitt (2005). \\
$\begin{array}{l}\text { Mayor status de salud, menor volumen de recursos } \\
\text { destinados a la atención de la salud, mayor volumen } \\
\text { de recursos destinado a actividades más productivas, } \\
\text { mayor crecimiento. }\end{array}$ & Van Zon y Muysken (2005). \\
\hline
\end{tabular}

Fuente: elaboración propia (2011). 
Se puede observar dos canales principales ampliamente identificados en la literatura, a través de los cuales el status de salud afecta al crecimiento económico, un canal directo y un canal indirecto. El canal directo se relaciona con la idea de que a mayor status de salud mayor productividad y de esta manera, mayor tasa de crecimiento de la economía.

Por su parte, el canal indirecto se vincula con que a mayor salud, menor tasa de depreciación del capital humano y mayor horizonte temporal de vida del individuo, generándose de esta forma una mayor inversión en capital humano y una mayor tasa de crecimiento de la economía.

Adicionalmente, existen otros canales que son identificados por algunos autores en relación a la influencia del status de salud sobre el crecimiento económico. Howitt (2005) plantea la idea de que un mayor status de salud aumenta la capacidad de aprendizaje $y$ de adaptabilidad a los cambios y la creatividad. Además de - al reducir la desigualdad- aumentar la asistencia a la escuela, generándose de esta forma un efecto positivo sobre el crecimiento. Por otro lado, Van Zon y Muysken (2005) afirman que, cuanto mayor sea el status de salud, menos recursos se destinarán a la atención de esta, los cuales quedarán disponibles para actividades más productivas, dando lugar de este modo a una mayor tasa de crecimiento.

Hasta el momento, se han presentado los diferentes canales con los que la salud afecta al crecimiento de la economía, lo cual permite observar la existencia de una estrecha relación entre el comportamiento del status de salud y el crecimiento económico. Esta estrecha relación puede considerarse también desde una perspectiva empírica, cuestión que se desarrolla en la siguiente sección.

\section{SALUD E INGRESO EN DISTINTAS REGIONES DEL MUNDO}

Para el análisis, se escogió un indicador representativo de cada una de las dimensiones que se quieren considerar, teniendo en cuenta que dadas las características de nuestro abordaje, en lugar de utilizar una variable representativa de crecimiento, se emplea una variable representativa de ingreso. En cuanto a salud, se eligió a uno de los principales indicadores utilizados en la literatura, que es la Tasa de Mortalidad Infantil ${ }^{1}$ (TMI) (London, Temporelli y Monterubbianesi, 2009). Respecto a ingreso, se tomó en cuenta al Producto Interno Bruto (PIB) per cápita medido de acuerdo a la Paridad del Poder Adquisitivo $(\mathrm{PPA})^{2}$, es decir, tomando en cuenta la capacidad de compra que tiene ese ingreso en los diferentes países, en base al nivel de precios en cada uno de ellos.

En relación a la base de datos utilizada, todos los datos corresponden al año 2009. Los datos de salud provienen de la base de datos del Banco Mundial, mientras que los datos de ingreso provienen de la Penn World Table (РWT). Una vez seleccionados los países para los cuales se cuenta con datos en las variables de interés, queda construida la muestra de 184 países representativos de todas las regiones del mundo.

De este modo, se presenta la situación relativa en cuanto a salud e ingreso que presentan las distintas regiones del mundo. La tabla $2^{3}$ presenta entonces el valor de los indicadores de salud e ingreso por continente. $\overline{1}$ La Tasa de Mortalidad Infantil representa el número de defunciones de niños menores de un año cada mil nacimientos vivos registrados.

2 La Paridad del Poder Adquisitivo (PPA) toma en cuenta el nivel de precios de cada país, de forma tal que permite medir de forma más real la capacidad de compra del ingreso.

3 Para el cálculo de los indicadores de cada región, se promedió el valor del indicador en cada país ponderado por la población de cada uno de ellos. 
TABLA 2

INDICADORES DE SALUD E INGRESO POR REGIÓN

2009

\begin{tabular}{lcccc}
\hline REGIÓN & TMI & PIB P/C & $\begin{array}{c}\text { DESIGUALDAD } \\
\text { TMI }\end{array}$ & $\begin{array}{c}\text { DESIGUALDAD } \\
\text { PIB P/C }\end{array}$ \\
\hline América del Norte & 8,57 & 33516,46 & 2,80 & 3,53 \\
Europa & 8,92 & 23370,97 & 31,50 & 33,88 \\
Oceanía & 12,68 & 30429,66 & 11,75 & 20,59 \\
América del Sur & 16,55 & 9023,73 & 5,25 & 3,24 \\
América Central y el Caribe & 24,26 & 7486,07 & 14 & 21,51 \\
Asia & 30,71 & 6460,80 & 51,5 & 137,78 \\
África & 64,74 & 2325,74 & 9,50 & 167,34 \\
\hline
\end{tabular}

Fuente: Elaboración propia con base en datos del Banco Mundial y de la PWT.

Del análisis de la tabla 2, se puede destacar que existe una estrecha relación entre el valor de los indicadores de salud e ingreso en las diferentes regiones. Las regiones que ocupan los tres primeros puestos en cuanto a indicadores de salud, también los ocupan en cuanto a indicadores de ingreso, mientras que los continentes que se encuentran en los últimos cuatro lugares en cuanto a salud, también lo hacen en cuanto a ingreso.

Adicionalmente, se consideran las cuestiones de desigualdad entre las diferentes regiones $y$ dentro de los distintos continentes. Respecto a la desigualdad entre regiones, en la primera columna se observa que la TMI en América del Norte es de 8,57 cada mil nacimientos, mientras que en África es de 64,74. Para dimensionar esta desigualdad entre las regiones $y$ teniendo en cuenta que la TMI se mide cada mil nacimientos, puede afirmarse que mueren más niños en África cada 150 nacimientos $(9,71)$ que en América del Norte cada 1000 nacimientos $(8,57)$. Por su parte, en cuanto al PIB per cápita, se observa que el PIB promedio en América del Norte es 14,5 veces el PIB promedio en África, lo que implica que con el ingreso que vive un individuo en América del Norte durante un día, debe vivir un individuo en África durante aproximadamente 15 días.

Finalmente, en relación a la desigualdad dentro las diferentes regiones del mundo, la misma puede observarse en las últimas dos columnas de la tabla 1. Para considerar la inequidad, se tomó el valor del primero y del último país, en cuanto a cada indicador y se obtiene el cociente. De este modo, se observa que la mayor desigualdad en cuanto a indicadores de salud, se da en Asia, donde el valor de los países con valores de TMI menos favorables, es 51 veces el valor del indicador de salud para el país líder de la región. Por otro lado, en América del Norte, el valor de TMI en México es el triple que el de Canadá, de forma tal que en esta región es donde se observa la menor desigualdad, en cuanto a indicadores de salud. Por su parte, en lo relativo a la desigualdad en el ingreso, se repite esta tendencia, en el sentido que la mayor desigualdad ocurre en Asia y la menor desigualdad en América del Norte. La gran desigualdad en Asia se debe a la amplitud del continente, con regiones densamente pobladas, en el cual conviven países ricos con altos indicadores de salud e ingreso como Japón, Singapur y Qatar, y por otro lado, países muy pobres y con indicadores de salud desfavorables como India y Pakistán.

\section{SALUD E INGRESO EN LOS DIFERENTES PAÍSES DEL MUNDO}

En esta sección se presenta la situación de los diferentes países del mundo, en cuanto 
a indicadores de salud e ingreso, analizando posteriormente el grado de asociación entre estas variables mediante la técnica de agrupamiento por tramos. La tabla 3 muestra el ranking de países seleccionados en cuanto a Tasa de Mortalidad Infantil (TмI).

TABLA 3

RANKING DE PAÍSES EN CUANTO A TASA DE MORTALIDAD INFANTIL PAÍSES SELECCIONADOS, 2009

\begin{tabular}{ccc}
\hline POSICIÓN & PAÍS & TMI \\
\hline 1 & Finlandia & 2 \\
1 & Islandia & 2 \\
1 & Japón & 2 \\
1 & Luxemburgo & 2 \\
1 & Singapur & 2 \\
1 & Eslovenia & 2 \\
1 & Suecia & 2 \\
50 & Bahréin & 9 \\
61 & Argentina & 12 \\
71 & México & 71 \\
100 & Paraguay & 21 \\
150 & Yemen & 57 \\
180 & Afganistán & 103 \\
181 & Rep. Cen. & 106 \\
182 & Africana & 108 \\
183 & Somalía & 112 \\
184 & Congo & 114 \\
\hline
\end{tabular}

Fuente: Elaboración propia a partir de los datos del Banco Mundial $y$ de la PWT.

Existe una serie de países (europeos y del sudeste asiático) que encabezan el ranking a nivel mundial, en cuanto a TMI, presentando 2 muertes cada mil nacidos vivos. Por otro lado, se observa un grupo de países africanos que cierran el ranking $y$ que presentan valores de TMI superiores a 100 muertes cada mil nacidos vivos, llegándose al extremo de Sierra Leona que presenta un valor del indicador de 114 . Al mismo tiempo, se puede analizar el grado de desigualdad en cuanto al valor de este indicador en el mundo. El valor de TMI en Sierra Leona es
57 veces mayor que el del grupo de países líderes. Es decir, que hay más muertes de menores de un año cada 20 nacimientos en Sierra Leona $(2,28)$ que en los países líderes cada 1000 nacimientos (2).

Por otro lado, la tabla 4 presenta el ranking de países seleccionados en cuanto a PIB per cápita. En este caso, se observa Qatar encabeza el ranking con un PIB per cápita de 159144 dólares. Los primeros puestos son ocupados nuevamente por países asiáticos y europeos. En los últimos puestos, nuevamente se observan los países africanos con un PIB per cápita menor a 500 dólares anuales. En el extremo aparece Zimbawe con un PIB per cápita de 142 dólares anuales.

TABLA 4

RANKING DE PAÍSES EN CUANTO A PIB PER CÁPITA - PPA

PAÍSES SELECCIONADOS, 2009

\begin{tabular}{ccc}
\hline POSICIÓN & PAÍ́S & PIB P/C (DÓLARES) \\
\hline 1 & Qatar & 159144 \\
2 & Luxemburgo & 84571 \\
3 & Emiratos Árabe & 52855 \\
4 & Noruega & 49974 \\
5 & Singapur & 47312 \\
50 & Palau & 14987 \\
60 & Argentina & 11959 \\
62 & México & 11633 \\
100 & El Salvador & 6341 \\
150 & Senegal & 1491 \\
163 & Somalía & 460 \\
164 & Liberia & 397 \\
165 & Burundi & 368 \\
166 & RD Congo & 231 \\
167 & Zimbawe & 142 \\
\hline
\end{tabular}

Fuente: Elaboración propia a partir de datos del Banco Mundial $y$ de la PWT.

En este caso, la desigualdad en cuanto a ingreso entre los distintos países del mundo puede verse claramente, dado que el ingreso en Qatar es 1120 veces el ingreso en Zimbawe. Es decir, con lo que vive una persona en Qatar durante un día, una persona en Zimbawe vive durante 3 años o lo que es lo mismo, viven 1120 personas durante un día en Zimbawe. 
Finalmente, se aplica la técnica de agrupamiento por tramos de forma tal de analizar si las posiciones relativas de los países -en cuanto a los indicadores de salud e ingresoson similares, es decir, si existe cierta interacción entre ambas dimensiones. La técnica de agrupamiento por tramos consiste en ordenar a los países de acuerdo al valor de determinado indicador y dividir al total de la población en tramos de igual número de países, en este caso en 10 tramos (4 de 19 países y 6 de 18 países). De esta forma, se identifica el tramo (desde el 1 hasta el 10) al que pertenece cada país y se analiza si pertenecen al mismo tramo. Si el tramo al que pertenecen los países en relación a ambas dimensiones coincide, esto significa que las posiciones relativas de los países en cuanto a los indicadores de salud e ingreso son similares, lo que implica que estas dimensiones se encuentran asociadas.

La técnica de agrupamiento por tramos brinda importante información respecto a la existencia de un movimiento conjunto o conducta asociada entre diferentes variables. Sin embargo, es necesario aclarar que presenta una limitación importante: no brinda información respecto a la causalidad entre las variables. Si queremos analizar las relaciones de causalidad, será necesario el empleo de técnicas econométricas, cuestión que excede los alcances del presente trabajo.

La tabla 5 muestra entonces el resultado de la aplicación de la técnica de agrupamiento por tramos. La primera columna indica la diferencia entre los tramos a los que pertenece cada país, en cuanto a salud e ingreso. Se puede observar que de los 184 países de la muestra, 60 están en el mismo tramo, tanto en cuanto a salud como a ingreso, mientras que 71 están en el tramo inmediatamente anterior o posterior. Es decir, aproximadamente el $71 \%$ de los países pertenecen al mismo tramo o al tramo más próximo en cuanto a salud e ingreso, mostrán-
TABLA 5

RESULTADOS DE LA TÉCNICA DE AGRUPAMIENTO POR TRAMOS

VARIABLE DE SALUD: TASA DE MORTALIDAD

INFANTIL

2009

\begin{tabular}{ccc}
\hline $\begin{array}{c}\text { DIFERENCIA EN } \\
\text { LOS TRAMOS }\end{array}$ & $\begin{array}{c}\text { NÚMERO } \\
\text { DE PAÍSES }\end{array}$ & PORCENTAJE \\
\hline 0 & 60 & 32,61 \\
1 & 71 & 38,59 \\
2 & 37 & 20,11 \\
3 o más & 16 & 8,69 \\
\hline
\end{tabular}

Fuente: Elaboración propia a partir de datos del Banco Mundial $y$ de la PWT.

dose de esta forma la fuerte interacción entre estas dimensiones.

Si se considera que la diferencia de tramos es 2, lo cual indica una posición de los países en ambas dimensiones relativamente similares, el porcentaje de países que se puede incluir sería de 91,31\%. Es decir, que más del 91\% de los 184 países considerados, presentan una situación similar en cuanto a los indicadores de salud y de ingreso escogidos. De esta forma, queda claramente establecido que existe una interacción considerable entre estas dimensiones.

Adicionalmente, para dar robustez a las conclusiones previamente mencionadas, se aplicó nuevamente la técnica de agrupamiento por tramos, empleando un indicador de salud diferente. En lugar de utilizar la Tasa de Mortalidad Infantil, usamos la Esperanza de Vida al Nacer. Para ello se utilizaron nuevamente, datos provenientes del Banco Mundial y teniendo en cuenta la disponibilidad de los mismos, el análisis se realiza para 178 países. La tabla 6 muestra los resultados de la técnica de agrupamiento por tramos empleando el PBI per cápita (PPA) y la Esperanza de Vida. 
TABLA 6

RESULTADOS DE LA TÉCNICA DE AGRUPAMIENTO POR TRAMOS

VARIABLE DE SALUD: ESPERANZA DE VIDA 2009

\begin{tabular}{ccc}
\hline $\begin{array}{c}\text { DIFERENCIA EN } \\
\text { LOS TRAMOS }\end{array}$ & $\begin{array}{c}\text { NÚMERO } \\
\text { DE PAÍSES }\end{array}$ & PORCENTAJE \\
\hline 0 & 55 & 30,90 \\
1 & 72 & 40,45 \\
2 & 28 & 15,73 \\
3 o más & 23 & 12,92 \\
\hline
\end{tabular}

Fuente: Elaboración propia a partir de datos del Banco Mundial $y$ de la PWT.

Los resultados obtenidos son similares a los que se obtuvieron al utilizar la Tasa de Mortalidad Infantil como indicador de salud. Es decir, de los 178 países de la muestra, 55 están en el mismo tramo, tanto en relación a salud como a ingreso, mientras que 72 están en el tramo inmediatamente anterior o posterior, en cuanto a estas dimensiones. Nuevamente, el $71 \%$ de los países pertenecen al mismo tramo o al tramo más próximo en cuanto a salud que en cuanto a ingreso.

Si se quiere considerar cuando la diferencia de tramos es 2 , el porcentaje de países que se podrían incluir se eleva a un $87 \%$. Es decir, que independientemente del indicador de salud que se utilice, es posible observar la estrecha relación entre salud e ingreso.

\section{CONCLUSIONES}

En el presente artículo se analizó cómo diferentes autores han desarrollado contribuciones en forma tal de incorporar a la salud dentro de la teoría del crecimiento económico como un determinante fundamental del mismo. Este análisis permitió identificar diferentes canales de influencia del status de salud sobre el crecimiento.

Así, se encontraron dos canales principales: un canal directo $y$ un canal indirecto, ampliamente identificados en la literatura, a través de los cuales el status de salud afecta al crecimiento económico. El canal directo se relaciona con la idea de que a mayor status de salud, mayor productividad. El indirecto se vincula con que a mayor salud, menor tasa de depreciación del capital humano y mayor horizonte temporal de vida del individuo, generándose de esta forma una mayor inversión en capital humano. Además de estos canales principales, existen canales secundarios vinculados con aspectos, tales como: la creatividad, la capacidad de aprendizaje $y$ de adaptación a los cambios, la desigualdad y la asignación de recursos entre diferentes actividades productivas.

El análisis teórico realizado muestra la estrecha vinculación existente entre el status de salud $y$ el nivel de ingreso. Este movimiento conjunto de las variables puede verificarse también mediante herramientas de estadística descriptiva. De este modo, se realizó un análisis para conocer la situación relativa de las distintas regiones y países del mundo en lo referente a indicadores de salud e ingreso, aplicándose además la técnica de agrupamiento por tramos, con la finalidad de conocer la similitud de la posición relativa de los países, en cuanto a estas dimensiones.

Del análisis efectuado, surge la existencia de una gran desigualdad inter e intra regiones, que en algunos casos llegan a puntos extremos. América del Norte, Europa y Oceanía aparecen claramente como las regiones del mundo con mejor valor de los indicadores de salud e ingreso, mientras que África es claramente el peor continente en lo relativo a estas dimensiones. Además, se observa que mientras Asia es la región más desigual, en cuanto a indicadores de salud e ingreso, América del Norte es la región menos desigual en estos aspectos. 
Por su lado, el análisis de agrupamiento por tramos muestra que el $91 \%$ de los países pertenece al mismo tramo o a un tramo próximo, en cuanto a indicadores de salud e ingreso, mostrándose claramente la estrecha relación que existe entre el status salud y el crecimiento económico.

\section{BIBLIOGRAFÍA}

Barro, R. Health and Economic Growth. PanAmerican Health Organization, 1991.

Ehrlich, I. y Lui, F. "Intergenerational trade, longevity and economic growth". Journal of Political Economy 99 (5). 1991: 1029-1059.

Kalemli-Ozcan, S.; Ryder, H. y Weil, D. "Mortality decline, human capital investment, and economic growth". Journal of Development Economics 62 (1). 2000: 1-23.

Howitt, P. Health, human capital and economic growth: a schumpeterian perspective. Pan-American Health Organization, 2005.

London, S.; Temporelli, K. y Monterubbianesi, P. D. "Vinculaciones entre salud, ingreso y educación: un análisis comparativo para América Latina”. Economía y Sociedad 14 (23). 2009: 125-146.

Monterubbianesi, P. D. "Una aproximación al análisis económico de la relación entre salud y crecimiento". Anales de la XLV Reunión Anual de la Asociación Argentina de Economía Política. 2010.

Monterubbianesi, P. D. "Un análisis teórico y estadístico sobre la relación entre salud y crecimiento". Actas del V Congreso Nacional de Estudiantes de Postgrado en Economía. 2011.

Lucas, R. "On the mechanics of development plannings". Journal of Monetary Economics 22 (1). 1988: 3-42.

Center for International Comparisons at the University of Pensylvania. "Penn World Table: production, income and prices". En: <http://pwt.econ.upenn.edu/> [consultado el 20 de febrero de 2012].

The World Bank. En: <http://www.worldbank. org/> [consultado el 20 de febrero de 2012].

Van Zon, A. y Muysken, J. "Health as a principal determinant of economic growth". Health and economic growth: findings and policy implications. G. LópezCasanovas, B. Rivera y L. Currais (eds.). The MIT Press, 2005.

Fecha de ingreso: 19/03/2013 Fecha de aprobación: 02/09/2013 